Supporting Information

\title{
The First Sequential Reaction Promoted by Manganese: Complete \\ Stereoselective Synthesis of $(E)$ - $\alpha, \beta$-Unsaturated Esters from 2,2-Dichloroesters and Aldehydes
}

\author{
José M. Concellón, * Humberto Rodríguez-Solla, Pamela Díaz and Ricardo Llavona \\ Departamento de Química Orgánica e Inorgánica, Facultad \\ de Química, Universidad de Oviedo, Julián Clavería, 8, \\ 33071 Oviedo, Spain. \\ email:jmcg@uniovi.es
}

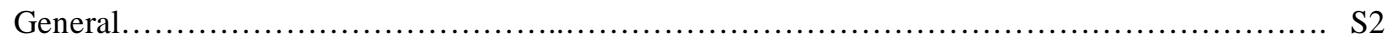

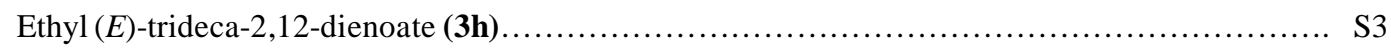

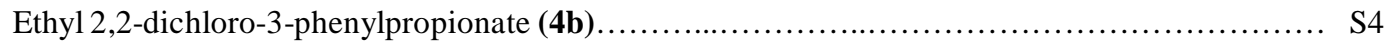

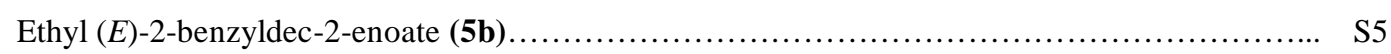

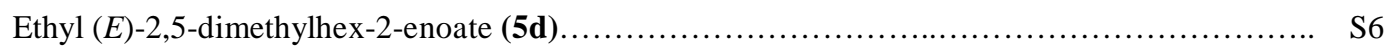

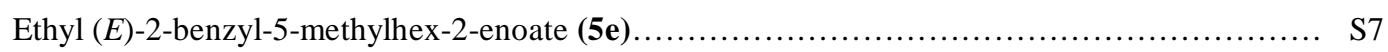




\section{GENERAL:}

Reactions requiring an inert atmosphere were conducted under dry nitrogen, and the glassware was oven dried $\left(120^{\circ} \mathrm{C}\right)$. THF was distilled from sodium/benzophenone ketyl immediately prior to use. All reagents were purchased in the higher quality available and were used without further purification. Flash column chromatography was carried out on silica gel 230-400 mesh. Compounds were visualized on analytical thin layer chromatograms (TLC) by UV light $(254 \mathrm{~nm}) .{ }^{1} \mathrm{H}$ NMR spectra were recorded at 200 , 300 or $400 \mathrm{MHz} .{ }^{13} \mathrm{C}$ NMR spectra and DEPT experiments were determined at 50 or $75 \mathrm{MHz}$. Chemical shifts are given in ppm relative to tetramethylsilane (TMS), which is used as an internal standard, and coupling constants $(J)$ are reported in Hz. The diastereoisomeric ratios were obtained using ${ }^{1} \mathrm{H}-\mathrm{NMR}$ analysis and GC-MS of crude products. GC-MS and HRMS were measured at $70 \mathrm{eV}$ or using FAB conditions. When HRMS could not be measured on molecular ion the HRMS of a significant fragment is given. Only the most important IR absortions $\left(\mathrm{cm}^{-1}\right)$ and the molecular ions and/or base peaks in MS are given. 
${ }^{1} \mathrm{H}$ and ${ }^{13} \mathrm{C}$ NMR spectra of products $3 \mathrm{~h}, 4 \mathrm{~b}, 5 \mathrm{~b}, 5 \mathrm{~d}$ and $5 \mathrm{e}$ :<smiles>C=CCCCCCCCC/C=C/C(=O)OCC</smiles>

(3h)

${ }^{1} \mathrm{H}$ NMR (300 MHz)

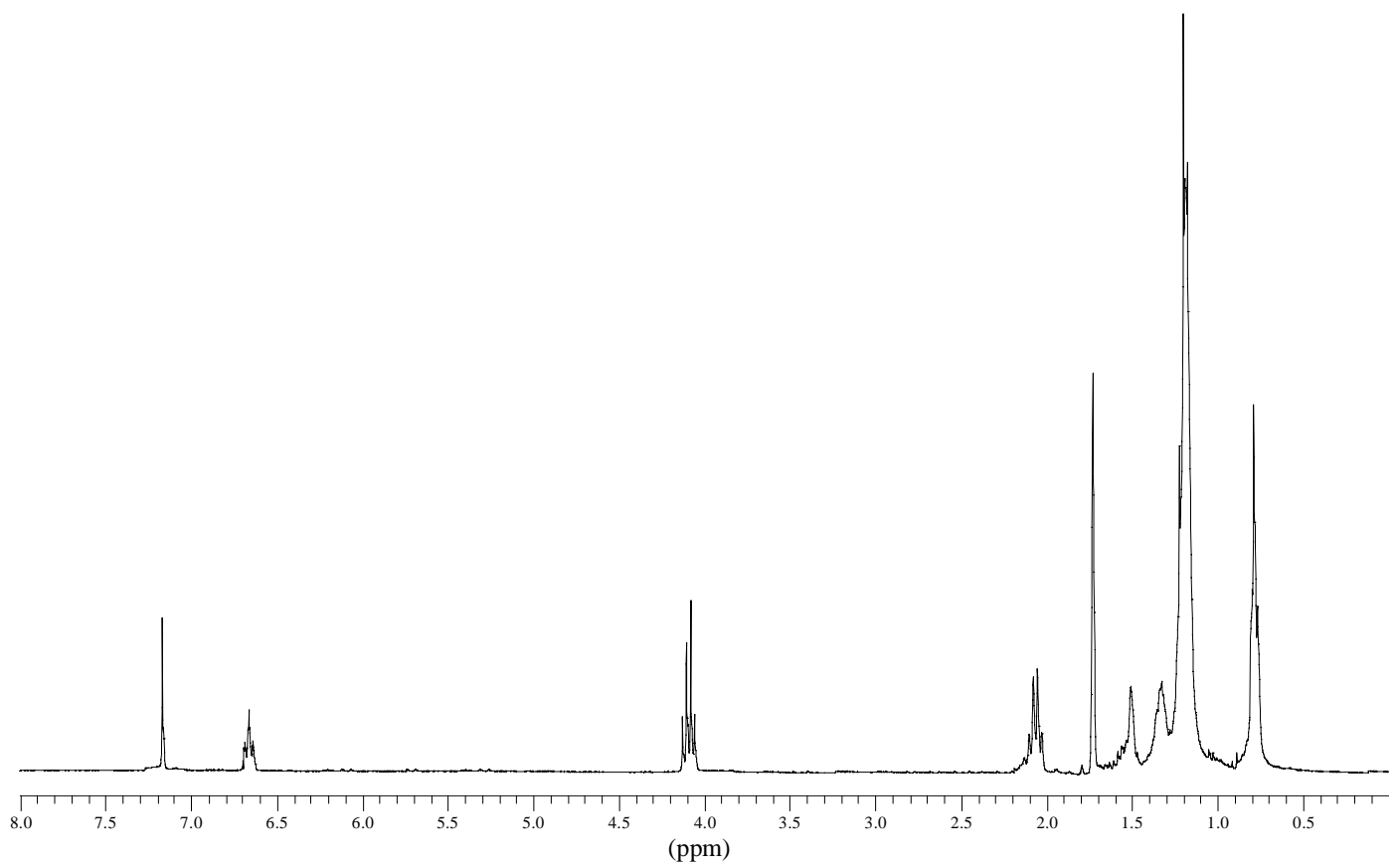

${ }^{13} \mathrm{C}$ NMR (300 MHz)

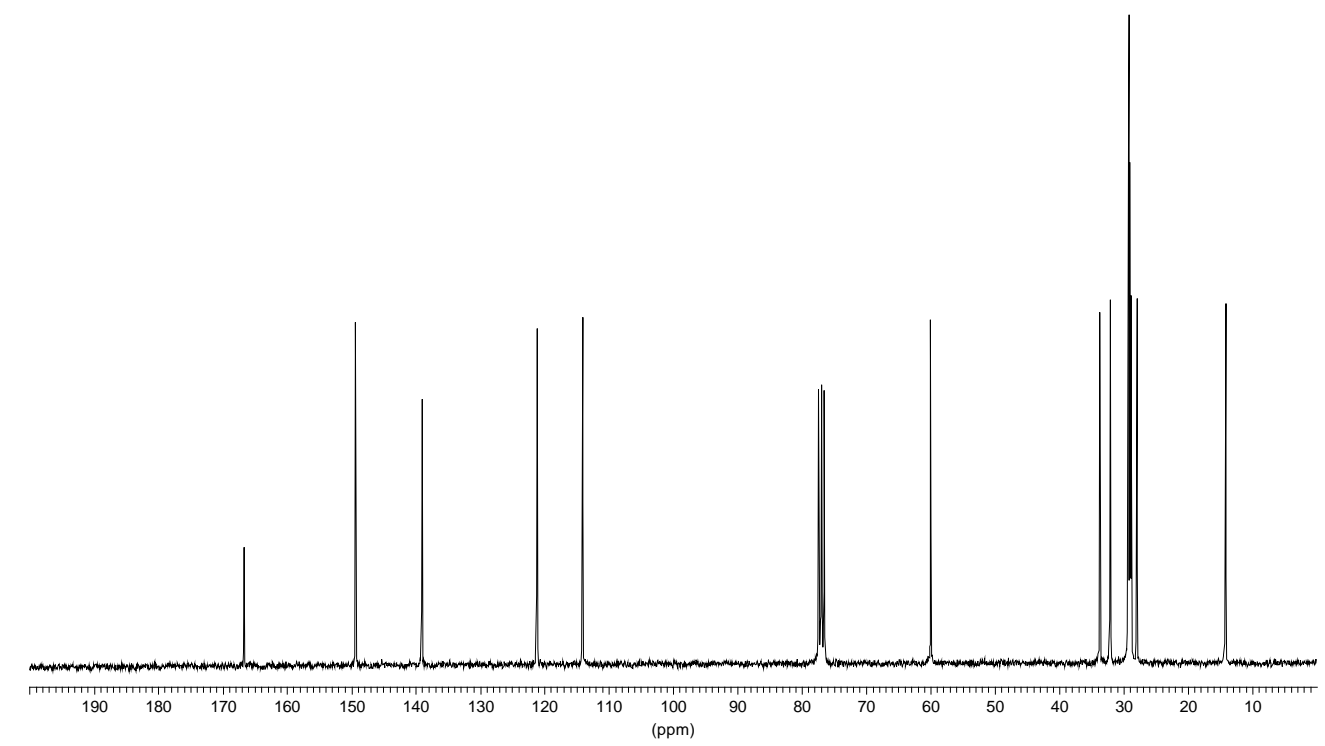


<smiles>CCOC(=O)C(Cl)(Cl)Cc1ccccc1</smiles>

(4b)

${ }^{1} \mathrm{H}$ NMR (300 MHz)

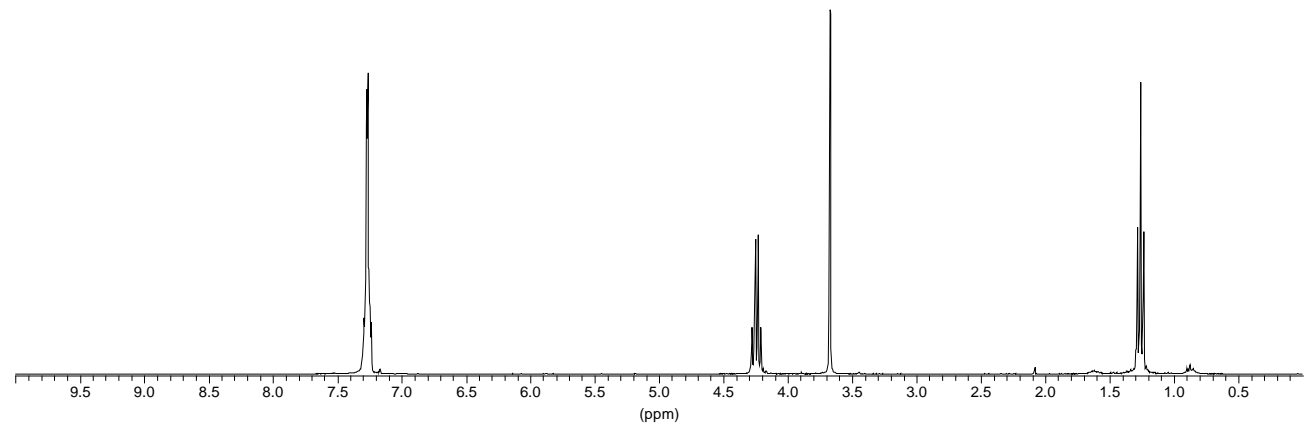

${ }^{13} \mathrm{C}$ NMR (300 MHz)

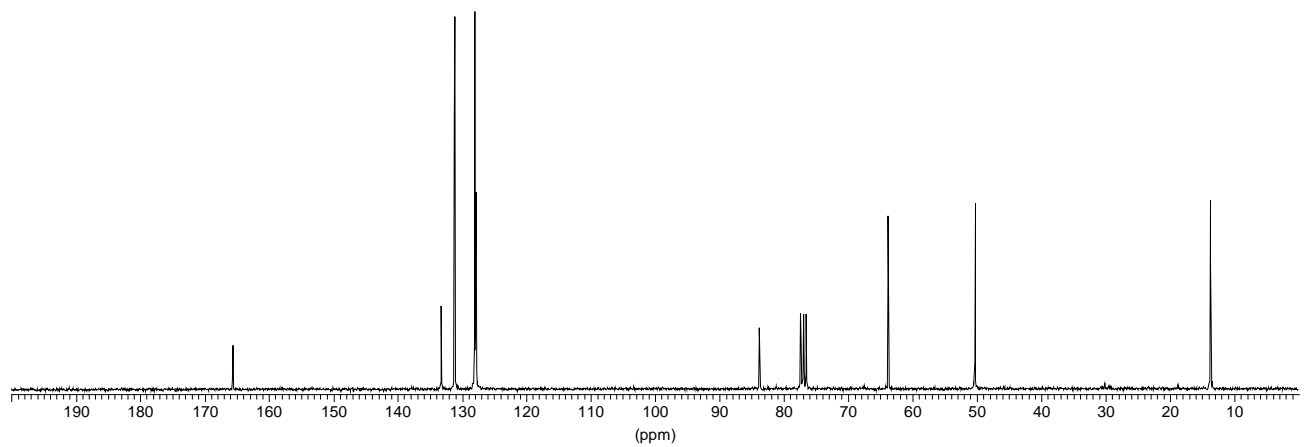




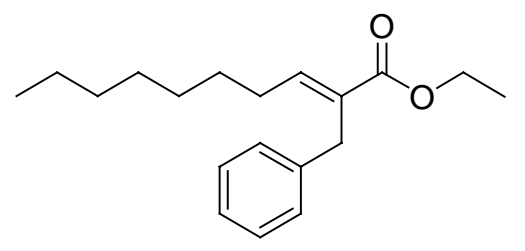

(5b)

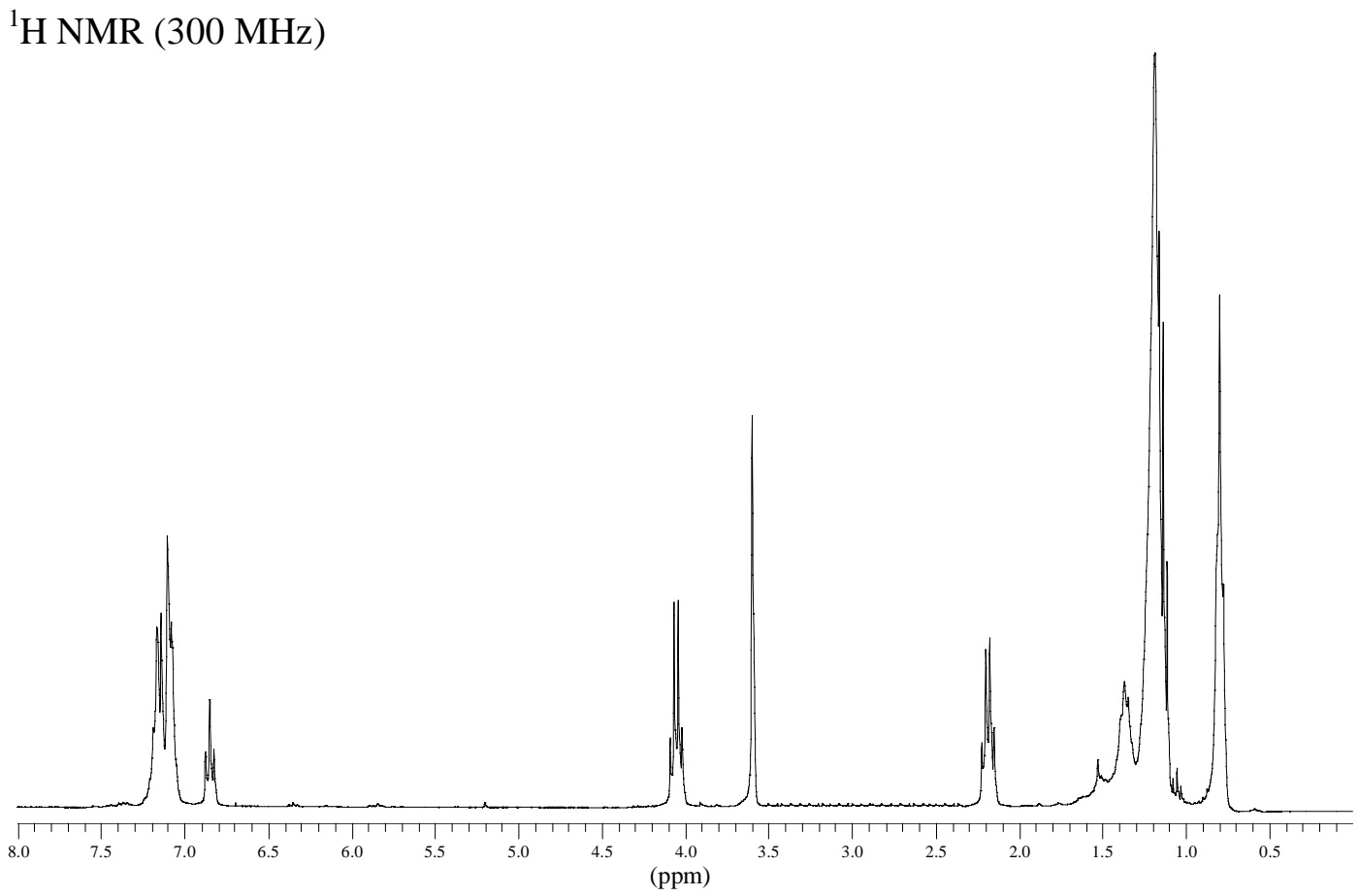

${ }^{13} \mathrm{C}$ NMR (300 MHz)

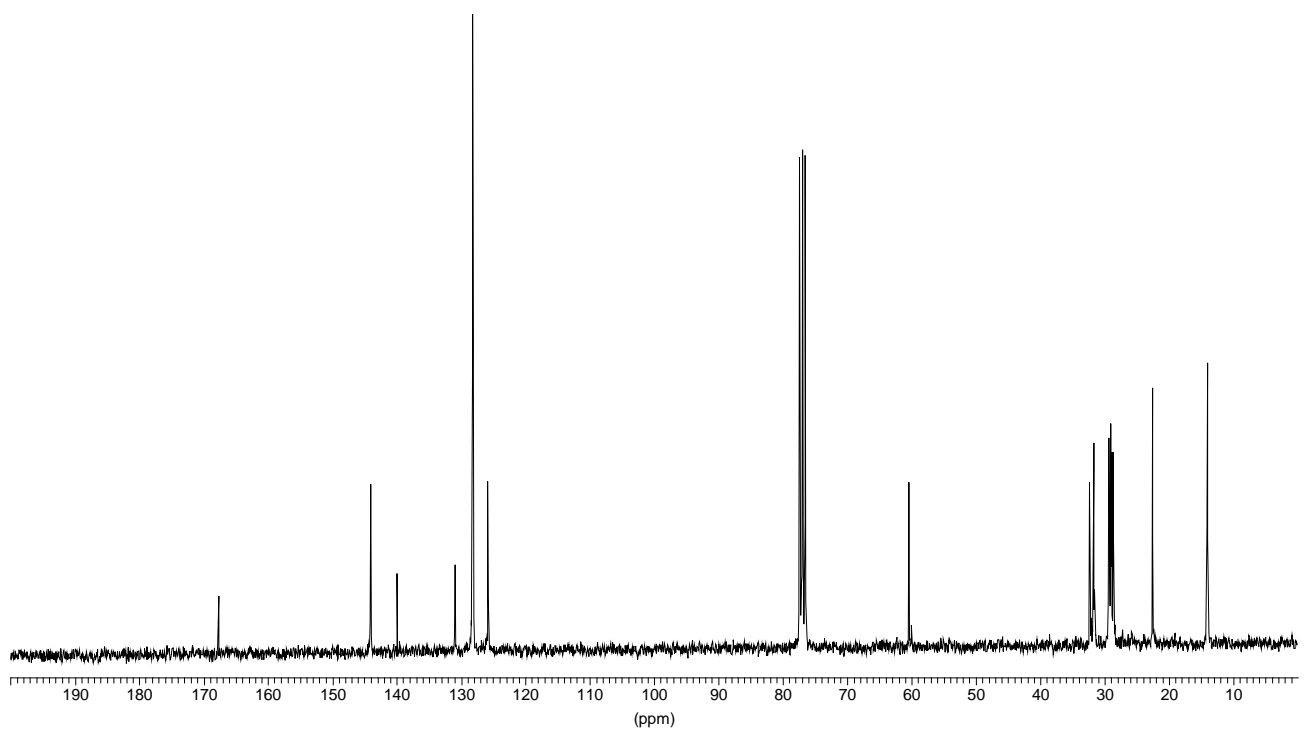




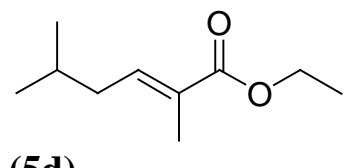

(Fd)

${ }^{1} \mathrm{H}$ NMR (300 MHz)

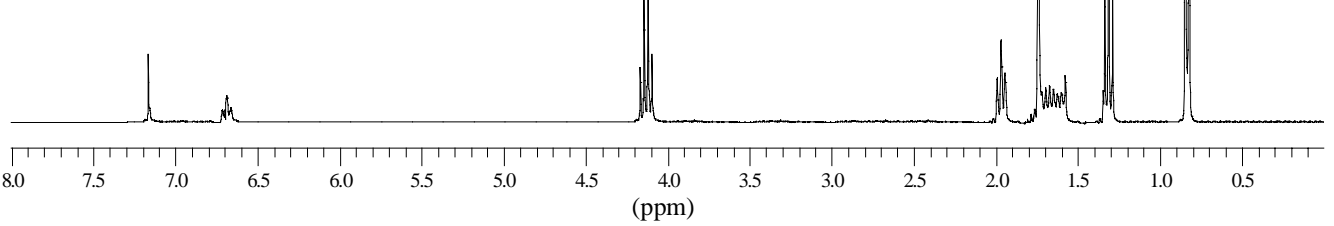

${ }^{13} \mathrm{C}$ NMR (300 MHz)

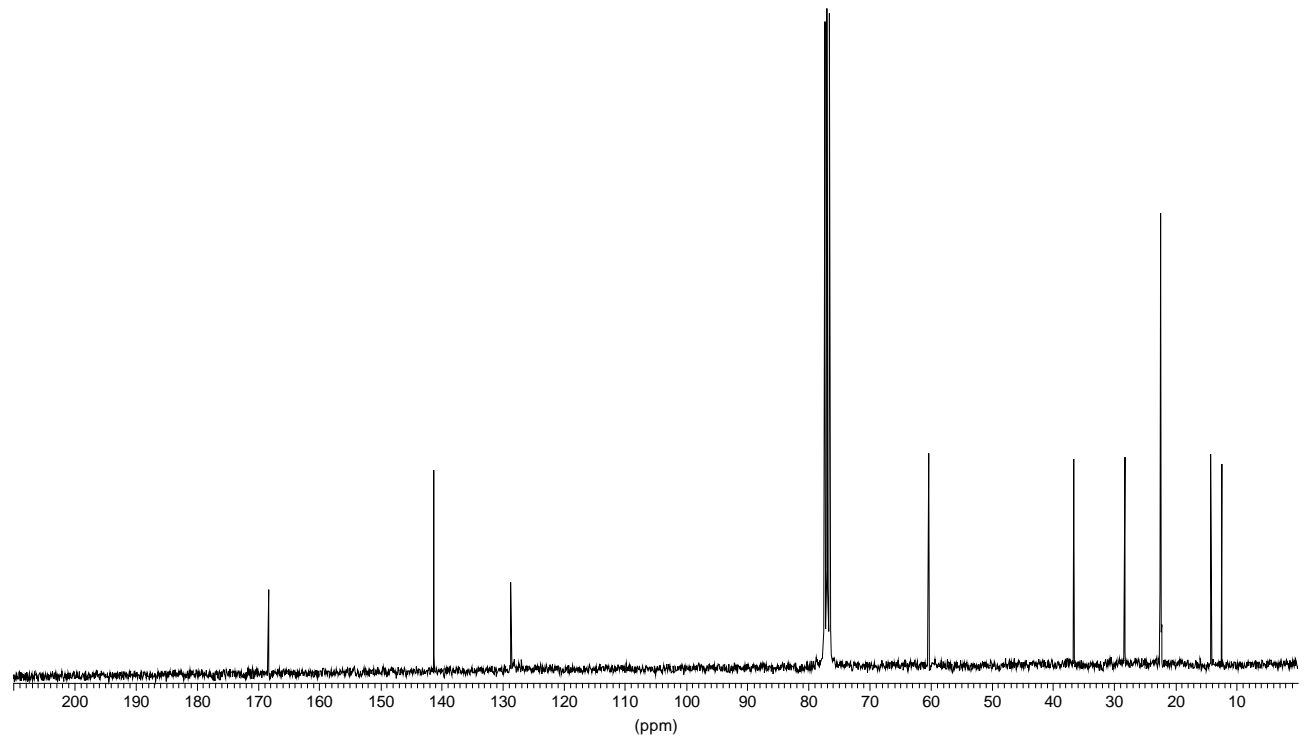

Sb 


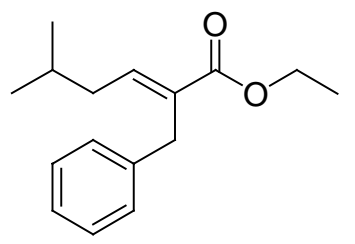

(5e)

${ }^{1} \mathrm{H}$ NMR (300 MHz)

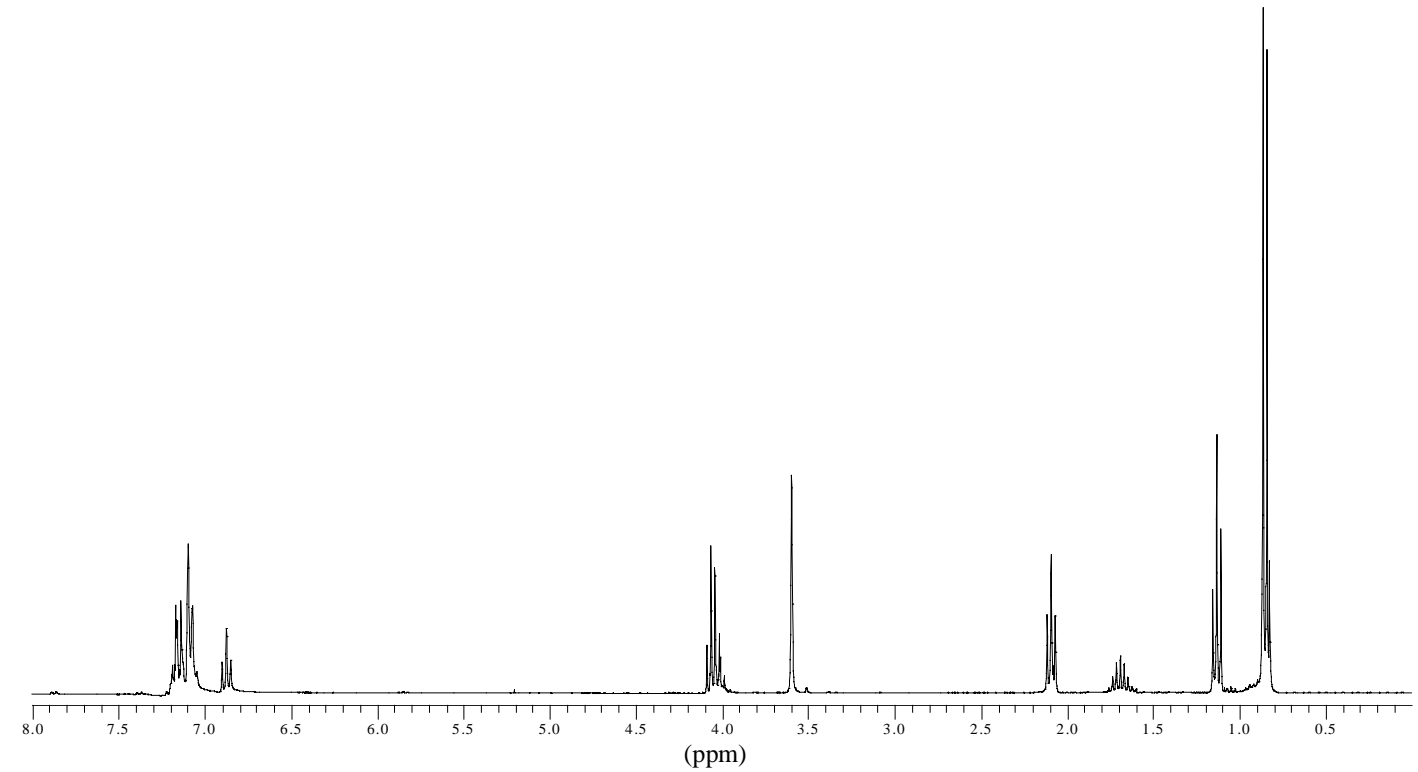

${ }^{13} \mathrm{C}$ NMR (300 MHz)

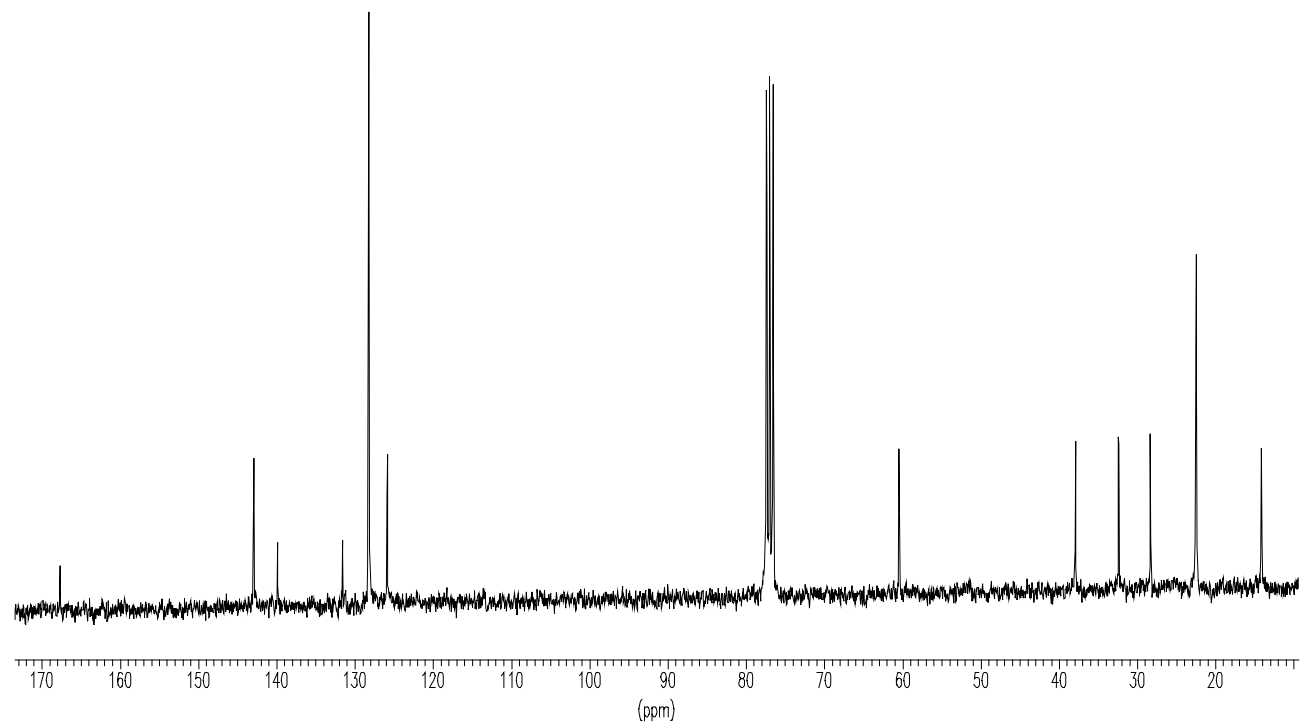

